\title{
Modelo 3C de Colaboração aplicado ao uso de um repositório para o desenvolvimento de interfaces para autistas
}

\author{
Áurea Hiléia da Silva Melo ${ }^{1,2,3}$, Clara Aben-Athar Bentes Fernandes', \\ Marco Santarelle da Silva Jardim ${ }^{1}$, Raimundo da Silva Barreto ${ }^{2}$ \\ ${ }^{1}$ Centro Universitário do Norte - Laureate International Universities (UNINORTE) \\ Caixa Postal 69010-060 - Manaus - AM - Brazil \\ ${ }^{2}$ Instituto de Computação - Universidade Federal do Amazonas (UFAM) \\ Caixa Postal 69.077-000 - Manaus - AM - Brasil. \\ ${ }^{3}$ Universidade do Estado do Amazonas - Escola Superior de Tecnologia (EST-UEA) \\ Caixa Postal 69050-020 - Manaus - AM - Brasil. \\ \{claraaabf, santarelle\}@unn.edu.br, \\ \{aurea.melo, rbarreto\}@icomp.ufam.edu.br
}

\begin{abstract}
New applications are developed day by day with the aim of helping children with autism in their therapies. In order to contribute to the increase of the production of these applications, this article presents a tool as a collaborative repository to guide developers of this type of application, following the good practices referring to interfaces for autistic users. This tool follows the Collaboration Model 3C, highlighting the value of the elements in their aspects and characteristics of communication, coordination and cooperation, so that they are complementary and make collaboration more and more effective.
\end{abstract}

Resumo. A cada dia novos aplicativos são desenvolvidos com o intuito de auxiliar crianças com autismo em suas terapias. De forma a contribuir com o aumento da produção desses aplicativos, este artigo apresenta uma ferramenta em forma de repositório colaborativo que possa servir de guia para os desenvolvedores desse tipo de aplicação, seguindo boas práticas relacionadas a interfaces destinadas a usuários autistas. Tal ferramenta, permite que o Modelo $3 C$ de Colaboração seja aplicado, ressaltando a valorização dos elementos em seus aspectos e características de comunicação, coordenação e cooperação, de modo que sejam complementares e torne a colaboração cada vez mais efetiva.

\section{Introdução}

O Autismo, também conhecido como Transtornos do Espectro Autista (TEA), é um transtorno que causa problemas no desenvolvimento da comunicação, socialização e comportamento [American Psychiatric Association 2013]. Esse transtorno atinge tanto crianças como adultos que apresentam o diagnóstico tardio, ao redor do mundo.

De acordo com as áreas do desenvolvimento humano afetadas, é possível destacar os principais fatores que definem os sintomas, tais como, dificuldades de 
comunicação, comportamentos repetitivos e mudanças sociais. Buscando diminuir essas barreiras, surgem as intervenções tecnológicas [Alessandrini, Cappelletti e Zancanaro 2014], que utilizam a tecnologia a seu favor, com o uso de software, jogos, ferramentas, entre outros recursos, para tornar o tratamento mais dinâmico, além de reduzir tempo e custos.

Automatizar determinadas operações por meio da intervenção tecnológica permite um tratamento mais eficaz, no entanto, é preciso observar as melhores práticas na concepção, elaboração e desenvolvimento de projetos, de forma a oferecer as devidas vantagens no tratamento do comportamento autista. Dessa forma, diversos trabalhos têm sido realizados para auxiliar no desenvolvimento desses recursos, entre eles, o processo ProAut (PROcesso para Projeto de Interfaces Destinadas a Usuários AUTistas) que surgiu como uma proposta para guiar equipes de desenvolvimento visando a construção desse tipo de produtos [Melo, Barreto e Conte 2016].

O ProAut é constituído de duas etapas: Design Inicial de protótipos e Avaliação e Refinamento do Protótipo, sendo que estas podem ser guiadas por um conjunto de diretrizes/recomendações. A cada ciclo de aplicação do processo, a equipe de desenvolvimento poderá utilizar o repositório de recomendações como guia de suporte a escolha das boas práticas. Da mesma maneira que o usuário tem a opção de consumir as informações contidas no repositório, ele também poderá disponibilizá-las, por meio das lições aprendidas pela equipe durante o desenvolvimento de um produto destinado a autistas, de modo que seja utilizado por eles ou por outros usuários em projetos futuros. O principal diferencial desse processo, segundo Melo, Barreto e Conte (2016), é fornecer "um conjunto de diretrizes e recomendações que podem ser usadas por equipes de desenvolvimento de forma a facilitar a construção de interfaces por meio da prototipação".

Este artigo propõe um repositório colaborativo para a disponibilização das diretrizes, no âmbito do desenvolvimento de projeto de interfaces destinadas a usuários autistas, o "Guidelines ProAut". Esta ferramenta permite a inserção, a busca e o feedback sobre as recomendações, através de comentários e avaliação das recomendações (concordar ou não concordar). O usuário, seja desenvolvedor ou especialista, ao compartilhar o conhecimento adquirido durante o desenvolvimento de um projeto, contribuirá para o aperfeiçoamento das práticas, e aumento de precisão e consistência sobre a experiência compartilhada.

Após a implementação do repositório, fez-se a análise para verificar se é dado o suporte adequado à colaboração, baseado no Modelo $3 \mathrm{C}$ de Colaboração, exercendo as três atividades fundamentais para a colaboração - comunicação, coordenação e cooperação, de modo que seja aplicado ao uso da plataforma. Foi realizado um levantamento de quais funcionalidades atendem a cada elemento do modelo $3 \mathrm{C}$ e quais ferramentas correspondem as funções apresentadas para compor um sistema colaborativo.

Este artigo está organizado da seguinte forma: a Seção 2 apresenta a fundamentação teórica, com uma breve explanação sobre Sistemas Colaborativos, para explorar mais detalhadamente o Modelo 3C de Colaboração, e em seguida fazer uma análise de trabalhos correlatos de acordo com objetivo deste artigo. A Seção 3 apresenta a ferramenta desenvolvida. Já a Seção 4, relata a abordagem de uso dos repositórios no 
que diz respeito a atuação dos elementos de colaboração. Por fim, na Seção 5, os resultados são analisados e as considerações finais e trabalhos futuros são apresentados.

\section{Fundamentação Teórica e Trabalhos Correlatos}

O conceito de colaboração refere-se a não somente interagir ou trocar informações, mas também a um ambiente com objetivos comuns e compartilhados, em que os participantes trabalhem juntos, com o propósito de contribuir para garantir o sucesso na execução de uma atividade [Gerosa 2006].

Nesse contexto, sistemas colaborativos são sistemas que apoiam a colaboração entre seus usuários e que se popularizaram a partir da estrutura de conexão entre os computadores em rede. Segundo a concepção de Nicolaci-da-Costa e Pimentel (2011), um sistema colaborativo constitui-se por um ciberespaço concebido para ser habitado, com um espaço para as interações humanas condizentes com os ideais das novas gerações para a realização do trabalho em grupo e que desejam colaborar, interagir e compartilhar, favorecendo o novo ser humano digital e a nova organização social.

A partir da análise de Pimentel et al. (2006), observou-se que a classificação dos sistemas colaborativos define-se a partir da taxonomia espaço-tempo e ao nível de aplicação. Dessa forma, as ferramentas colaborativas são classificadas de acordo com o lugar e o tempo das interações, referindo-se ao fato de estar à distância e de estabelecer uma comunicação síncrona ou assíncrona, que ora depende do estado de sincronia entre o emissor e o receptor, e ora é independente desse estado entre as partes envolvidas.

Visto que esse tipo de aplicação classifica-se de diversas formas, contudo ainda em consonância com Pimentel et al. (2006), considera-se que o Modelo 3C de Colaboração seja frequentemente usado pela literatura para classificar os sistemas colaborativos. Este modelo analisa a colaboração em três dimensões, representadas por: comunicação, coordenação e cooperação, de modo que é determinada a separação em dimensões para focar nos aspectos relevantes para a análise. Para Fuks et al. (2011), mesmo que algumas ferramentas disponham mais características de um elemento do que de outro, estas conterão mecanismos para dar suporte aos $3 \mathrm{C}$ 's, pois os três são interdependentes e se inter-relacionam para que haja a colaboração.

Nos subitens a seguir, serão explorados separadamente os elementos do Modelo $3 \mathrm{C}$ e apresentados os trabalhos relacionados com essa pesquisa, buscando expor tanto a arquitetura quanto as funcionalidades ligadas a um repositório de recomendações.

\subsection{Modelo 3C de Colaboração}

O Modelo 3C de Colaboração abrange a comunicação, coordenação e cooperação. Sendo que a comunicação envolve a troca de mensagens, voltada exclusivamente para a ação dos compromissos. Enquanto que por meio da coordenação, as pessoas, as atividades e os recursos são gerenciados para lidar com conflitos e se organizarem, evitando o desperdício de comunicação e dos esforços de cooperação. Por fim, a cooperação é caracterizada pela execução das tarefas por meio da operação conjunta no espaço compartilhado, gerando e manipulando objetos ou informações para a sua produção. Durante a concretização dos 3 C's, informações são geradas e disponibilizadas através de elementos de percepção [Gerosa, Fuks e Lucena 2003]. 
Para fins de análise, os elementos serão explorados de maneira isolada para focar nos seus aspectos individuais, mesmo que estejam associados para a geração da colaboração. Por essa razão, este isolamento ocorre apenas para a melhor compreensão sobre como ocorre a aplicação de cada componente nos sistemas colaborativos.

Comunicação é a ação de transmitir e receber mensagens, para que haja o entendimento comum entre as partes envolvidas (comum-ação), com trocas de ideias e negociações para a tomada de decisão e realização dos compromissos propostos, neste caso, na ocorrência da cooperação [Fuks et al. 2011]. Este processo de transmissão se realiza de maneira síncrona ou assíncrona, diferenciando-se conforme o tempo de resposta entre as partes.

Existem ferramentas que apoiam os dois modos de comunicação, de forma complementar ou independente. Algumas ferramentas possibilitam o envio e recebimento instantâneo de mensagens, de maneira que os envolvidos permaneçam em um estado de sincronia, tendo como exemplo as videoconferências. Enquanto que a outra classe de ferramentas, oferece suporte para fluxos de mensagens assíncronas, que não necessitam de um tempo de resposta imediato, como o e-mail, por exemplo. Os fóruns também são considerados ferramentas assíncronas, mesmo que seja um transmissor se comunicando com vários receptores.

Coordenação é o elemento que orienta e organiza para o atendimento dos compromissos gerados pela demanda do elemento de comunicação. De forma pontual, são definidos objetivos e restrições que determinarão quando as tarefas serão realizadas, na ordem e no tempo previstos. Portanto, é a ação de ordenar em conjunto: pessoas, tarefas e recursos (co-ordem-ação) [Fuks et al. 2011]. Além de oferecer suporte aos demais elementos, evitando conflitos em detrimento de falhas na comunicação e cooperação, mantendo-os alinhados às suas responsabilidades.

Essa dimensão fornece um tipo de política de acesso e um conjunto de regras para manter a ordem no ambiente compartilhado. Em geral, assemelha-se a estrutura lógica de uma plataforma. Utilizando o exemplo de uma típica sala de bate-papo, constitui-se num suporte à coordenação a exibição da lista de participantes, indicando os que estão conectados e disponíveis para a conversação [Pimentel et al. 2006]. Por isso, sem coordenação os esforços da comunicação e cooperação estariam comprometidos.

Cooperação é a produção conjunta das partes envolvidas, com a finalidade de que as mesmas trabalhem juntas para realizar as atividades. Isto posto, é a ação de operar em conjunto no espaço compartilhado (co-operar-ação) [Fuks et al. 2011]. Ao executarem as tarefas, no ambiente proposto e com objetivos definidos, os interessados responsabilizam-se por transformar, produzindo ou modificando de maneira compartilhada.

As atividades executadas durante o processo desse elemento, estabelecem a necessidade de comunicação e, de forma encadeada, demandam coordenação para orientar e guiar na realização das novas tarefas. Um exemplo disso, é o registro e compartilhamento como funcionalidades de uma ferramenta, tal qual o chat, a área que disponibiliza o registro das mensagens publicadas se constitui num suporte à cooperação [Pimentel et al. 2006]. 
Em consoante ao exposto, o modelo $3 \mathrm{C}$ foi esquematizado conforme ilustra a Figura 1, representando as definições e a maneira como os elementos se integram, evidenciando o inter-relacionamento entre eles para que a colaboração ocorra. No centro do diagrama, tem-se a percepção apontando através da seta bidirecional para as três dimensões, identificando que o grupo de pessoas é responsável por estimular e intermediar as propriedades. Com isso, segundo Gerosa, Fuks e Lucena (2003), por meio da percepção das atividades haverá informações suficientes para auxiliar na sincronização, orientando-se em torno de seus contextos individuais.

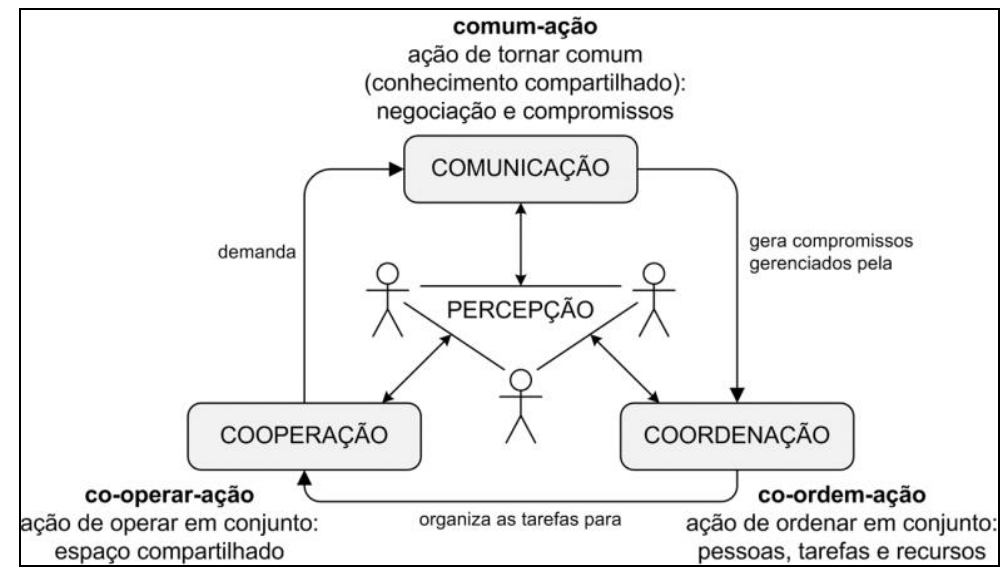

\section{Figura 1. Modelo 3C de Colaboração} Fonte: Fuks et al. (2011)

\subsection{Trabalhos Correlatos}

A partir da abordagem do Modelo 3C de Colaboração, observou-se que para obter a classificação de sistema colaborativo é necessária a comunicação, coordenação e cooperação em função do Modelo 3C [Pimentel et al. 2006].

Na Figura 2 é apresentado como os sistemas são posicionados no espaço triangular constituído por três seções, de modo que o grau de suporte a cada uma das dimensões interfere no seu posicionamento, cujos elementos da colaboração estão nos vértices. Reafirmando que apesar da disposição da classe de aplicações apontar mais para uma dimensão, identificando o seu objetivo principal e oferecendo maior suporte a um determinado $\mathrm{C}$, também é preciso dar suporte para os outros dois elementos.

Dentre as classes nomeadas, o "espaço de informação compartilhada" será o foco das ferramentas correlatas aqui mencionadas, devido à assimilação com o repositório Guidelines ProAut.

Silva et al. (2016), propõem uma plataforma web colaborativa denominada ColabAD, um sistema colaborativo de áudio-descrição que possibilita aos usuários compartilhar imagens utilizando a tecnologia assistiva (recursos e serviços que ampliam as habilidades funcionais de pessoas com deficiência) da Áudio-Descrição (AD). Em que uma imagem específica é descrita e detalhada em formato de texto, para a compreensão de pessoas com deficiência visual. Análoga aos Q\&A's Systems (Questions and Answers Systems) ou Sistemas de Perguntas e Respostas, que Furtado e Andrade (2011) definem como sistemas com a finalidade de encontrar respostas (answers) para problemas, colocados em formato de perguntas (questions) por usuários que interagem de modo colaborativo. 


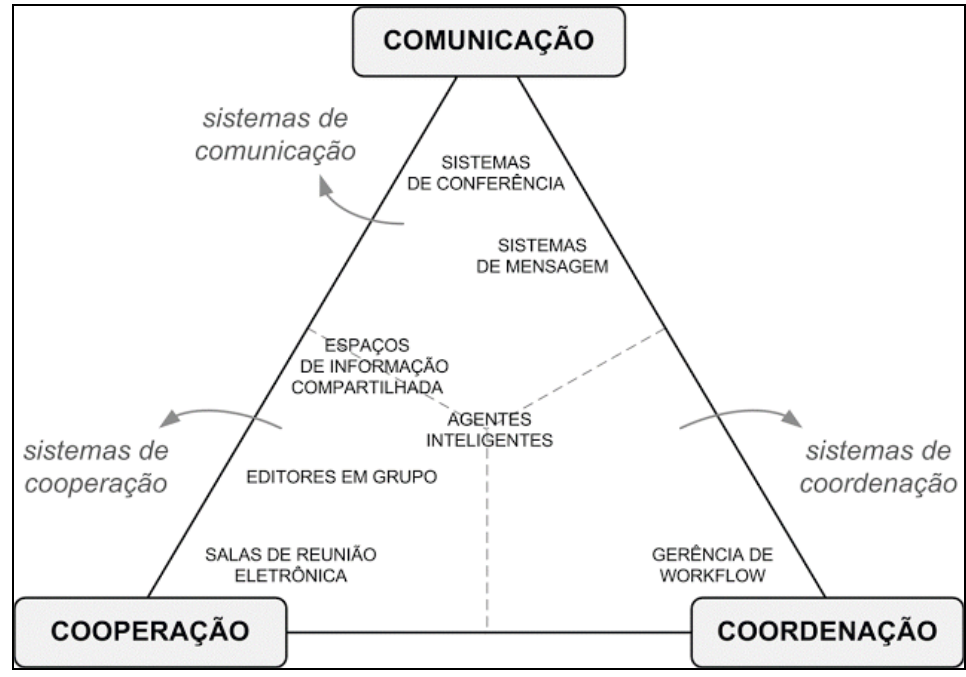

Figura 2. Classificação 3C dos sistemas colaborativos Fonte: Pimentel et al. 2006

Assim como Q\&A's Systems, o ColabAD apresenta uma estrutura a qual os usuários com perfil comum geram demanda de imagens para que os usuários especializados realizem a AD das imagens. Posteriormente, para então torná-las públicas no repositório, é preciso que um usuário revisor o avalie. Com isso, verificouse que apesar de estar associada a uma plataforma de apoio à acessibilidade por meio da $\mathrm{AD}$, esta não corresponde diretamente a ideia principal do Guidelines ProAut, que fornece um repositório de recomendações.

Outra ferramenta com o objetivo que se assemelha, é a AccessibilityUtil.com, uma ferramenta de colaboração de experiências em acessibilidade na Web, onde é possível "criar artefatos, visualizar os já presentes e fazer avaliações, inserindo suas experiências e decisões de projeto, contribuindo com relatos de uso e técnicas para se ter a completude dos critérios de acessibilidade para cada artefato" [Bittar et al. 2011]. Esse repositório tem como objetivo, disponibilizar práticas de acessibilidade advindas da experiência de desenvolvedores, focando nas boas práticas de ambiente Web, relacionando com as diretrizes de acessibilidade do World Wide Web Consortium (W3C), apresentada em Web Content Accessibility Guidelines (WCAG) 2.0.

Semelhante ao Guidelines ProAut, o AccessibilityUtil possibilita a interação entre os usuários, que podem adicionar as recomendações e/ou artefatos, visualizar as já cadastradas e fazer comentários avaliando quanto aos critérios da WCAG 2.0, de maneira que possam contribuir para complementar as diretrizes de acessibilidade. Diferente e restritamente a proposta apresentada neste artigo, a ferramenta deste estudo foca nas recomendações para projeto de interfaces destinadas a usuários autistas.

Dentre os trabalhos relacionados, o projeto GAIA (Guia de Acessibilidade de Interfaces Web com foco em aspectos do Autismo) de Britto e Pizzolato (2016) é o mais similar com o objetivo do ProAut. Embora a proposta do guia esteja relacionada a um repositório com o objetivo de prover um conjunto de diretrizes de design adequados às necessidades de crianças com autismo, este apenas publica as recomendações, baseando-se nas sugestões de diretrizes coletadas que passaram por um processo de identificação, sistematização e consolidação das recomendações propostas. 
Portanto, o Guia de Acessibilidade - GAIA oferece somente uma coleção de recomendações e o seu processo difere do Guidelines ProAut, pois possui o formato e a estrutura de um blog, não estabelecendo a colaboração entre os usuários, visto que não existem funcionalidades que permitam a interação em sua plataforma. Diante disso, todas as postagens das diretrizes e seus artefatos são inseridos por administradores do site. Apesar de ter uma mediação na inclusão de contribuições ao repositório, dificultando a contribuição dos membros da comunidade, isso pode ser uma estratégia para manter a qualidade das informações disponibilizadas no repositório.

A partir dos trabalhos correlatos, percebe-se que há diferentes escopos e objetivos em cada estratégia proposta apresentada para o desenvolvimento com acessibilidade, buscando a melhoria de interfaces de produtos destinadas às pessoas que necessitam de alguma assistência.

Como observa Bittar et al. (2011), consideram-se que para as questões de acessibilidade, toda ferramenta que auxilie e forneça boas práticas para $o$ desenvolvimento é válida, basta extrair as contribuições oportunas para cada finalidade, como o ColabAD, com as imagens áudio-descritivas para cegos, o GAIA como um repositório estático, resultante de um estudo que propôs recomendações a partir de uma revisão sistemática e o AccessibilityUtil que apresenta boas práticas de ambiente Web, relacionando com as diretrizes de acessibilidade do W3C - WCAG 2.0.

Face ao exposto, a Seção 4 proporciona uma análise dessas ferramentas, juntamente com o Guidelines ProAut, para verificar conforme as funcionalidades definidas quais se adequam ao Modelo 3C de Colaboração.

\section{Guidelines ProAut}

O Guidelines ProAut é uma plataforma em formato de repositório para dar suporte as fases do desenvolvimento de interfaces direcionadas para usuários autistas utilizando o ProAut proposto por Melo, Barreto e Conte (2016). A ferramenta permite que os usuários inseriram suas recomendações com a finalidade de ganhar visibilidade $\mathrm{e}$ consistência, sobre as experiências obtidas durante as fases do ProAut, ou ainda, sobre as fontes de estudos publicadas em anais, revistas ou sites especializados. A seguir serão descritas a arquitetura do repositório e as suas funcionalidades.

\subsection{Arquitetura da ferramenta}

O repositório foi desenvolvido utilizando a linguagem de programação PHP juntamente com o framework Laravel, por apresentar uma documentação expressiva e rica em componentes, possibilitando o desenvolvimento ágil de todas as funcionalidades do projeto [Laravel 2017]. O projeto foi criado com base no padrão de desenvolvimento MVC (Model-View-Controller), sabendo que o Laravel possui esse padrão nativo e o mantém atualizado para fornecer a melhor experiência de desenvolvimento em sua utilização.

O MVC é um padrão arquitetural que divide a aplicação em três camadas: modelo, visão e controlador, respectivamente representado pelo gerenciamento dos dados, pela estrutura visual e pelo controle que interpreta e mapeia as ações do usuário. Desse modo, na camada de visão, foi utilizado o framework Bootstrap que torna ágil a construção de layouts para web, por meio das linguagens HTML, CSS e Javascript, e 
por implementar seus componentes responsivos [Bootstrap 2017]. Permitindo ao Guidelines ProAut tornar-se adaptável a qualquer formato de dispositivo, tais como, computadores, smartphones e tablets, desde que tenham suporte aos navegadores (browser).

O sistema de gerenciamento de banco de dados escolhido para o projeto foi o MySQL, capaz de responder de forma rápida a demanda de consulta, cadastro, alteração e remoção de dados necessária na aplicação. Em consentimento, a equipe modelou o banco de dados para permitir que os usuários atuem de forma autônoma, inserindo as recomendações sem que seja preciso tratá-las por mediadores da plataforma, como mostra a última versão da modelagem representada pela Figura 3.

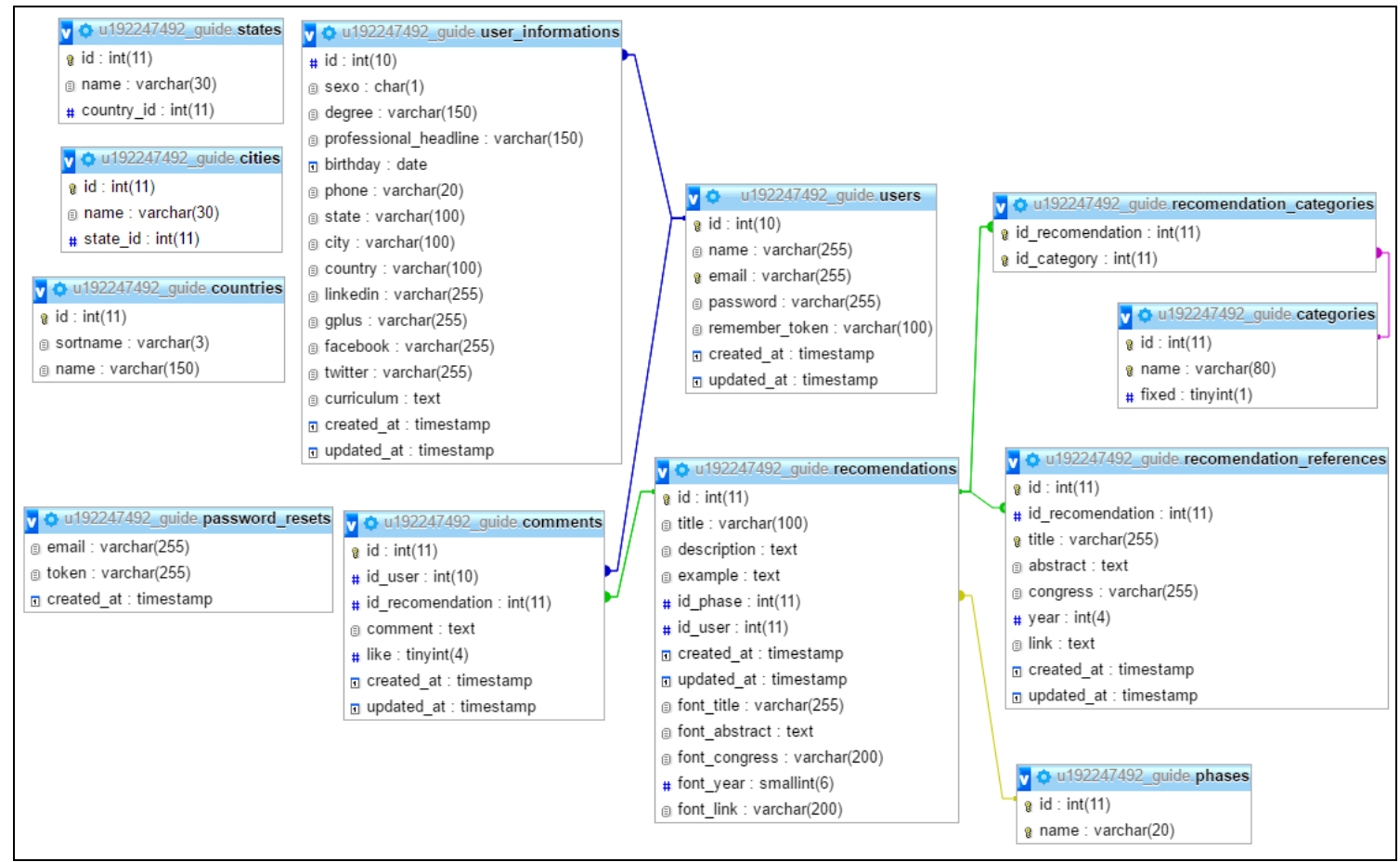

Figura 3. Modelo de dados relacional da ferramenta

A categoria, a fase e os comentários são os elementos primordiais para a classificação das recomendações, com a finalidade de mostrar em que etapa se aplica, quais as categorias que se enquadram e como está sua posição na classificação dos resultados da pesquisa, por meio das opiniões formadas sobre as recomendações.

\subsection{Características e Funcionalidades da ferramenta}

A ideia para o desenvolvimento da ferramenta partiu da concepção do ProAut, processo que fornece um conjunto de diretrizes/recomendações que surge a partir das experiências adquiridas pela equipe, durante o desenvolvimento de interfaces para quaisquer produtos de software cujo seja utilizado por autistas. O objetivo da plataforma é, portanto, servir de guia e sistema colaborativo para auxiliar equipes de desenvolvimento, visando a utilização das boas práticas fornecidas pelo repositório.

Considera-se um guia, o fornecimento de um conjunto de recomendações para ajudar profissionais a desenvolver interfaces seguindo as experiências compartilhadas pelos usuários. Quanto ao fato de ser colaborativo, dá-se em decorrência de tornar o 
repositório um ambiente com espaço de informação compartilhada, permitindo que quaisquer usuários insiram as recomendações de acessibilidade para autistas, desde que atendam aos critérios de cadastro, com o preenchimento completo das informações.

Esse repositório conta com funcionalidades para manter o perfil do usuário e as diretrizes. Ambos os cadastros são detalhados para apresentar a plataforma com maior grau de seriedade. Para isso, os dados mantidos do usuário referem-se ao seu perfil, e informações como data de nascimento, sexo, escolaridade, profissão e redes sociais, são guardadas. Para a diretriz são exigidos os dados que a caracterizam e também sobre a(s) sua(s) referência(s).

Cada recomendação permite que sejam inseridas uma ou mais referências, para mostrar que possui embasamento para a sua disponibilização. Informações sobre o título da diretriz, fase, categoria(s), descrição e exemplo de uma aplicação concluem esta parte, enquanto dados como título da referência, resumo, anais ou revista, ano e link servem para certificar que a recomendação apresenta experiências com credibilidade, pois para a inserção é preciso obrigatoriamente informar estes campos.

Haverá sempre uma referência obrigatória para ser cadastrada para a diretriz, mesmo que esta seja baseada exclusivamente por experiências vividas e não publicadas, e somente neste caso, os campos sobre as referências poderão ser apontados para o perfil pessoal do responsável. De outra maneira, se houver a necessidade de incluir outras, é preciso acessar a tabela de exibição das recomendações, que fornece acesso para as cadastradas pelo próprio usuário, para realizar o cadastro de nova(s) referência(s).

Ao criar a recomendação, automaticamente esta ficará visível para todos os usuários, que poderão visualizá-la previamente através de uma lista (Figura 4). Após selecioná-la dentre as demais, será exibida com todas as informações, mostrando os detalhes sobre ela, como é evidenciado pela Figura 5, assim como o espaço para comentar, inserindo a opinião a respeito da diretriz selecionada.

Para realizar um comentário sobre a recomendação, é necessário que o usuário efetue o login. Essa contribuição aparecerá no formato de uma opinião, identificando se este concorda ou discorda. Por consequência, estas avaliações reforçam a colaboração na ferramenta, por expor as recomendações de quaisquer usuários e também por permitir receber opiniões de terceiros, reportando os diferentes pontos de vista de quem contribui e comenta através da plataforma.

O repositório, disponibiliza funcionalidades para otimizar o seu uso, como o cadastro simultâneo de múltiplas diretrizes, a partir de uma planilha, a pesquisa avançada e o download dos resultados da pesquisa. Para isso, estimando um melhor posicionamento desses resultados, foi utilizada como base a classificação ao comentar, para retornar nas primeiras posições da lista aqueles que tiverem mais classificações positivas. 


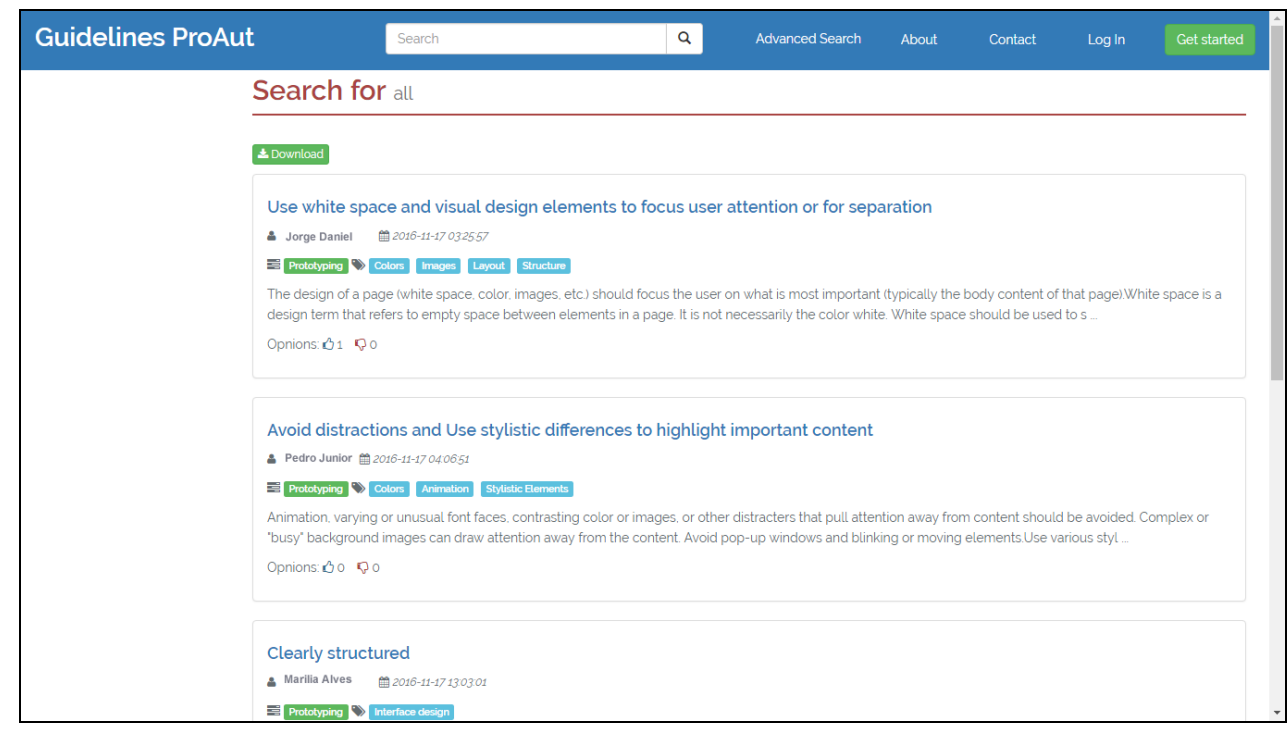

Figura 4. Lista de Recomendações disponíveis na plataforma

O Guidelines ProAut além de ser um repositório e disponibilizar as diretrizes/recomendações, também permite aos usuários contribuir por meio de comentários, fornecendo suas opiniões quanto as recomendações disponibilizadas. Esse conjunto de funcionalidades da ferramenta é focado no conceito da colaboração e segue as características dos elementos do Modelo 3C de Colaboração.

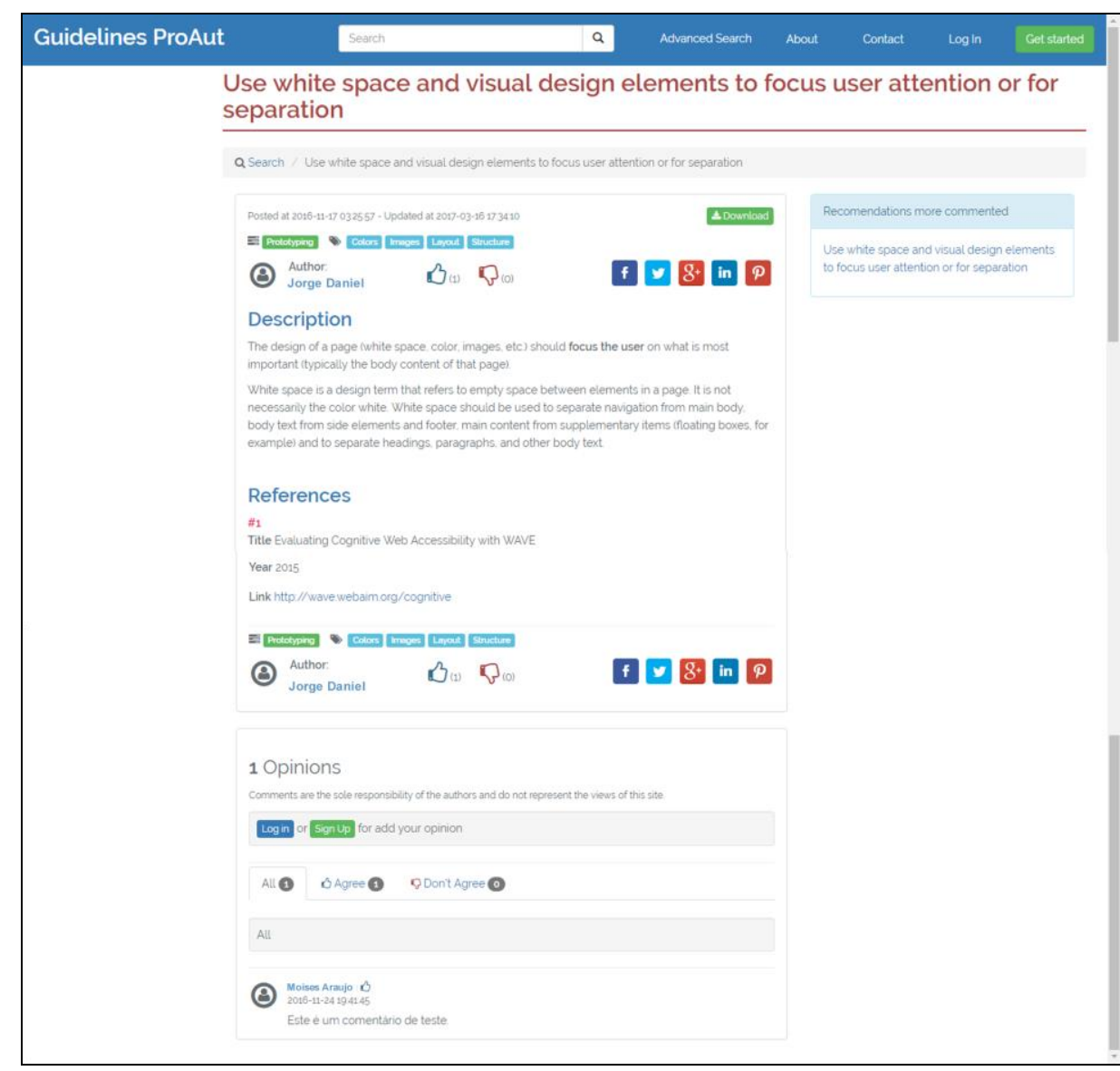

Figura 5. Exibição da Recomendação com um comentário (agree) de teste 
Inicialmente o repositório apresentará as recomendações inseridas a partir das pesquisas realizadas em Melo, Barreto e Conte (2016), que estarão disponíveis aos usuários para a coleta do feedback através dos comentários com as opiniões fornecidas ao concordar ou discordar sobre o que foi relatado como diretriz. A plataforma também permite a inserção de recomendações por quaisquer usuários devidamente cadastrados, que ao cadastrar uma diretriz com todas as informações obrigatórias, esta ficará disponível assim como as demais existentes, para os julgamentos realizados pela comunidade de usuários do Guidelines ProAut.

\section{Modelo 3C de Colaboração aplicado as ferramentas correlatas e ao Guidelines ProAut}

Como visto, comumente, o Modelo 3C de Colaboração é usado para classificar os Sistemas Colaborativos. Estes, por sua vez, integram um conjunto de funcionalidades que contribuem para comunicação, coordenação e cooperação. A partir disso, as funções essenciais dos repositórios foram aqui classificadas segundo o modelo de colaboração.

A Tabela 1 apresenta as funcionalidades com a sua abrangência nas ferramentas e conforme o enquadramento em um ou mais elementos que compõem o modelo $3 \mathrm{C}$. Funções tais como: criar conta e exibir perfil de usuário, cadastrar um objeto de colaboração pela plataforma ou muitos de uma vez, a partir de uma planilha, editar, listar e exibir objeto de colaboração, realizar comentário, demonstrando concordar ou discordar sobre o objeto de colaboração, realizar pesquisas simples e avançadas e, realizar o download dos resultados da pesquisa, também em formato de uma planilha.

Dentre as ferramentas analisadas, identificou-se que, somente a GAIA, não contém mecanismos para dar suporte aos três elementos. Pois, o projeto em questão, foca apenas em compartilhar as recomendações, servindo de guia para os profissionais que desejam saber sobre as diretrizes de acessibilidade para interfaces. Em vista disso, esta ferramenta somente atende a funcionalidade de "exibir objeto de colaboração", que apenas se enquadra na comunicação, não oferecendo suporte aos outros dois elementos.

As demais atenderam aos critérios do modelo colaborativo. Cada uma apresentando as funcionalidades que lhes eram pertinentes de acordo com os seus objetivos. Por exemplo, o ColabAD é uma plataforma que permite a atuação dos 3C's na função de "edição do objeto de colaboração", formando uma rede de colaboradores áudio-descritores, que permite a edição por usuários com perfis distintos em diferentes etapas, atuando de forma livre, porém de maneira comprometida em prover uma áudiodescrição de qualidade. De modo que ao final, seja publicada uma imagem que passou por identificação dos usuários, por meio de um processo efetuado por pessoas capacitadas para a realização da $\mathrm{AD}$ de imagens, buscando atender as expectativas dos usuários finais.

Nesse arcabouço de ferramentas, assim como o Guidelines ProAut, a que mais proporciona funcionalidades que consente com os elementos definidos pelo modelo $3 \mathrm{C}$, é a AccessibilityUtil, ao empregar o conceito de colaboração aplicado ao repositório, tendo as recomendações sendo de inteira responsabilidade dos usuários. Dessa maneira, os artefatos, objeto de colaboração assim denominados por eles, podem ser inseridos por quaisquer usuários que de modo colaborativo elaboram o conteúdo da ferramenta. Das funções listadas apenas as que atuam para otimizar o uso da ferramenta, não abrangem 
as funcionalidades do AccessibilityUtil, como a pesquisa avançada, o cadastro a partir de uma planilha, possibilitando a inserção de várias recomendações de uma vez, e o download dos resultados da pesquisa também em arquivo no formato de planilha.

Tabela 1. Tabela comparativa das funcionalidades classificadas de acordo com

o Modelo 3C de Colaboração

\begin{tabular}{|c|c|c|c|}
\hline Funcionalidades & COMUNICAÇÃO & COORDENAÇÃO & COOPERAÇÃO \\
\hline $\begin{array}{c}\text { Criar conta como } \\
\text { usuário }\end{array}$ & & $\begin{array}{l}\text { Guidelines ProAut; } \\
\text { ColabAD; } \\
\text { AccessibilityUtils }\end{array}$ & \\
\hline $\begin{array}{c}\text { Exibir Perfil de } \\
\text { usuário }\end{array}$ & & $\begin{array}{l}\text { Guidelines ProAut; } \\
\text { AccessibilityUtils }\end{array}$ & \\
\hline $\begin{array}{l}\text { Cadastrar Objeto de } \\
\text { Colaboração }\end{array}$ & $\begin{array}{l}\text { Guidelines ProAut; } \\
\text { ColabAD; } \\
\text { AcessibilityUtils }\end{array}$ & $\begin{array}{l}\text { Guidelines ProAut; } \\
\text { ColabAD; } \\
\text { AccessibilityUtils }\end{array}$ & \\
\hline $\begin{array}{l}\text { Cadastrar múltiplos } \\
\text { Objetos de } \\
\text { Colaboração a partir } \\
\text { de uma planilha. }\end{array}$ & Guidelines ProAut; & Guidelines ProAut; & \\
\hline $\begin{array}{l}\text { Editar Objeto de } \\
\text { Colaboração }\end{array}$ & $\begin{array}{l}\text { Guidelines ProAut; } \\
\text { ColabAD; }\end{array}$ & $\begin{array}{l}\text { Guidelines ProAut; } \\
\text { ColabAD; }\end{array}$ & ColabAD; \\
\hline $\begin{array}{l}\text { Listar Objetos de } \\
\text { Colaboração }\end{array}$ & & $\begin{array}{l}\text { Guidelines ProAut; } \\
\text { ColabAD; } \\
\text { AccessibilityUtils }\end{array}$ & $\begin{array}{l}\text { Guidelines ProAut; } \\
\text { ColabAD; } \\
\text { AccessibilityUtils }\end{array}$ \\
\hline $\begin{array}{l}\text { Exibir Objeto de } \\
\text { Colaboração }\end{array}$ & & $\begin{array}{l}\text { Guidelines ProAut; } \\
\text { ColabAD; } \\
\text { AccessibilityUtils; } \\
\text { GAIA }\end{array}$ & $\begin{array}{l}\text { Guidelines ProAut; } \\
\text { ColabAD; } \\
\text { AccessibilityUtils }\end{array}$ \\
\hline $\begin{array}{l}\text { Realizar comentários } \\
\text { sobre os Objetos de } \\
\text { Colaboração }\end{array}$ & $\begin{array}{l}\text { Guidelines ProAut; } \\
\text { AccessibilityUtils }\end{array}$ & $\begin{array}{l}\text { Guidelines ProAut; } \\
\text { AccessibilityUtils }\end{array}$ & $\begin{array}{l}\text { Guidelines ProAut; } \\
\text { AccessibilityUtils }\end{array}$ \\
\hline $\begin{array}{c}\text { Concordar ou } \\
\text { Discordar do Objeto } \\
\text { de Colaboração }\end{array}$ & $\begin{array}{l}\text { Guidelines ProAut; } \\
\text { AccessibilityUtils }\end{array}$ & $\begin{array}{l}\text { Guidelines ProAut; } \\
\text { AccessibilityUtils }\end{array}$ & $\begin{array}{l}\text { Guidelines ProAut; } \\
\text { AccessibilityUtils }\end{array}$ \\
\hline $\begin{array}{l}\text { Realizar pesquisas } \\
\text { simples }\end{array}$ & & $\begin{array}{l}\text { Guidelines ProAut; } \\
\text { AccessibilityUtils }\end{array}$ & \\
\hline $\begin{array}{c}\text { Realizar pesquisas } \\
\text { avançadas }\end{array}$ & & Guidelines ProAut; & \\
\hline $\begin{array}{l}\text { Download dos } \\
\text { resultados da pesquisa }\end{array}$ & & Guidelines ProAut; & \\
\hline
\end{tabular}


O repositório, foco deste artigo, apresenta como funcionalidades de comunicação, aquelas associadas a troca de informações. Neste caso em específico, foi aplicada somente de forma assíncrona, através do cadastro e edição de objetos de colaboração e da realização de comentários, concordando ou não sobre a diretriz. Casos específicos em quem as funções fornecem facilidades para permitir a troca de ideias, por meio das argumentações nas recomendações e das opiniões expostas.

Para a coordenação, todas as funcionalidades foram abrangidas, pois esta lida com a organização e o gerenciamento de toda a plataforma, atuando para garantir que as demais funções operem da maneira correta. Já para a cooperação, em que se destacam as operações realizadas em conjunto para a produção de conteúdo em um ambiente compartilhado, as funcionalidades compreendidas neste elemento são a lista e exibição dos objetos de colaboração, representados respectivamente pelas recomendações listadas a partir das pesquisas no repositório e pela apresentação da recomendação selecionada, que exibe ao final as opiniões a respeito da experiência levantada, identificando se os usuários concordam ou não concordam com a diretriz.

Observando a abordagem dos elementos presentes nas funcionalidades do repositório Guidelines ProAut, percebeu-se que o Modelo 3C de Colaboração ajuda a organizar e gerenciar os compromissos assumidos durante a interação entre os usuários e a realização do trabalho colaborativo através da soma dos trabalhos individuais, assegurando o entendimento e a negociação entre as partes envolvidas. Quando ao publicar uma recomendação, permitir que os usuários através da ferramenta tenham a opção de comentar e com isso expor suas opiniões quanto a diretriz em questão, para construir um entendimento comum, trocar ideias, discutir e aprender, como forma de alinhar e refinar as ideias, para a disseminação da experiência abordada.

\section{Conclusão e Trabalhos Futuros}

O Modelo 3C de Colaboração aplicado ao uso do Guidelines ProAut, visa oferecer suporte para que os usuários se tornem responsáveis ao contribuir com as suas experiências em formato de recomendações e, também, ao argumentar e dinamizar por meio de comentários, fornecendo diretrizes consistentes e consolidadas para guiar no uso das boas práticas para o desenvolvimento de produtos de software, com suporte para a acessibilidade de pessoas com autismo.

Dessa forma, o repositório auxilia o usuário ao exercer três atividades essenciais: comunicar-se, coordenar-se e cooperar, a partir dos recursos oferecidos no ambiente, guiando-os através da utilização das recomendações compartilhadas por outros usuários. Com isso, estes atuarão de forma colaborativa para disponibilizar diretrizes enquadradas nas fases de desenvolvimento do ProAut.

O repositório apresenta um espaço para expor além das diretrizes, as opiniões dos demais usuários, evidenciando que o foco não está em somente apresentá-las sem que haja a possibilidade de interações. Por isso, a ferramenta apresenta-se em formato colaborativo, de modo que preza pelas contribuições das experiências vivenciadas pelos usuários, permitindo com a devida permissão apresentar os diferentes pontos de vistas.

A colaboração traz resultados positivos tanto para os colaboradores ativos de conteúdo quanto para os visualizadores de experiências, pois o primeiro se beneficia por 
apresentar diretrizes buscando feedbacks, para ganhar visibilidade e consistência sobre elas, enquanto que o outro é favorecido por receber o apoio de recomendações resumidas e de fácil entendimento para a sua aplicação no processo de desenvolvimento.

Portanto, o objetivo deste trabalho é apresentar a aplicação do Modelo 3C ao uso do repositório Guidelines ProAut, influenciando o ambiente através da colaboração, ao oferecer suporte aos 3C's: comunicação, coordenação e cooperação. Evidenciando que dentro dos limites da liberdade de expressão é possível constituir uma base de recomendações sobre acessibilidade destinadas a autistas, escopo esse delimitado para melhor filtrar e se encaixar em um grupo dedicado e interessado por essa especialidade.

Como resultados parciais básicos foi aplicado um teste piloto com um profissional da área de Interação Humano Computador, que sugeriu melhorias no design da aplicação. De modo que o resultado da busca trouxesse informações das recomendações, quanto a fase na qual se encontra, as categorias relacionadas e a classificação, representadas pela contribuição dos usuários através dos comentários.

Para os trabalhos futuros pretende-se realizar testes de comunicabilidade e de usabilidade, de forma que se possa validar a ferramenta nesses âmbitos, buscando receber feedback sobre o design e o nível de satisfação do usuário, ao observar até que ponto há a facilidade de uso e a capacidade de compreensão, diante da interação. Além disso, uma extensão da ferramenta seria incluir a funcionalidade de indicação das recomendações mais adequadas, a partir dos parâmetros de características do projeto.

\section{Referências}

American Psychiatric Association. (2013). "DSM-5th: Diagnostic and Statistical Manual of Mental Disorders". 5th ed. American Psychiatric Press

Alessandrini, A., Cappelletti, A., and Zancanaro, M. (2014). "Audio-augmented paper for therapy and educational intervention for children with autistic spectrum disorder." In International Journal of Human-Computer Studies, 72(4), 422-430

Bittar, T. J.; Amaral, L. A.; Lobato, L. L. and Fortes, R. P. M. (2011). "AccessibiltyUtil.com: uma ferramenta para colaboração de experiências de acessibilidade na web". In Webmedia 2011 - Proceedings of the $17^{\text {th }}$ Brazilian Symposium on Multimedia and the Web. X Workshop on Tools and Applications.

Britto, T. C. P. and Pizzolato, E. B. (2016) "GAIA: uma proposta de um guia de recomendações de acessibilidade de interfaces Web com foco em aspectos do Autismo”. In Anais do XXVII Simpósio Brasileiro de Sistemas Colaborativos.

Bootstrap - The world's most popular mobile-first and responsive front-end framework. Disponível em: http://getbootstrap.com/. Acesso em: 28 de Fevereiro de 2017

Fuks, H.; Raposo, A.; Gerosa, M. A.; Pimentel, M.; Filippo, D. and Lucena, C. J. P. (2011) "Teorias e Modelos de Colaboração" In Pimentel, M. and Fuks, H. (Org.). Sistemas Colaborativos. Rio de Janeiro: Elsevier. cap. 2, p. 16-33.

Furtado, A., and Andrade, N. (2011) "Ativistas, passageiros, ocasionais e especialistas: Perfis de usuário na construção de um site de Q\&A". In Anais do VIII Simpósio Brasileiro de Sistemas Colaborativos. 
Gerosa, M. A. (2006) "Desenvolvimento de Groupware Componentizado com Base no Modelo 3C de Colaboração" Rio de Janeiro. 275p. Tese de Doutorado Departamento de Informática, Pontifícia Universidade Católica do Rio de Janeiro.

Gerosa, M. A., Fuks, H., and Lucena, C.J.P. (2003), "Suporte à Percepção em Ambientes de Aprendizagem Colaborativa", In Revista Brasileira de Informática na Educação, V. 11, No. 2, Setembro 2003, ISSN 1414-5685, Sociedade Brasileira de Computação, pp. 75-85.

Laravel - The PHP Framework For Web Artisans. Disponível em: https://aravel.com/. Acesso em: 28 de Fevereiro de 2017

Nicolaci-da-Costa, A. M. and Pimentel, M. (2011) "Sistemas Colaborativos para uma nova sociedade e um novo ser humano" In: Pimentel, M. and Fuks, H. (Org.). Sistemas Colaborativos. Rio de Janeiro: Elsevier. cap. 1, p. 3-15.

Melo, A. H. da S.; Barreto, R. and Conte, T. (2016) "ProAut: Um Processo para Apoio de Projetos de Interface de Produtos de Software para Crianças Autistas”. In Cadernos de Informática - Vol. 9, n. 1 (outubro. 2016). Porto Alegre: Instituto de Informática UFRGS, 2013. p. 27-41.

Pimentel, M.; Gerosa, M. A.; Filippo, D.; Raposo, A.; Fuks, H. and Lucena, C. J. P. (2006) "Modelo 3C de Colaboração no Desenvolvimento de Sistemas Colaborativos". In Anais do III Simpósio Brasileiro de Sistemas Colaborativos, Natal - RN, 20 a 22 de Novembro de 2006. ISBN 85-7669-097-7. Porto Alegre: SBC, 2006. p. 58-67.

Silva, J. F. da; Nora, T. D; Bertolini, C. and Gomes, J. (2016) "ColabAD: Sistema Colaborativo de Áudio-descrição" In: Anais do EATI - Encontro Anual de Tecnologia da Informação e STIN - Simpósio de Tecnologia da Informação da Região Noroeste do RS. Frederico Westphalen - RS. Ano 6 n. 1 p. 260-263. 